\title{
Glass Blowing on a Wafer Level
}

\author{
E. Jesper Eklund, Student Member, IEEE, and Andrei M. Shkel, Associate Member, IEEE
}

\begin{abstract}
A fabrication process for the simultaneous shaping of arrays of glass shells on a wafer level is introduced in this paper. The process is based on etching cavities in silicon, followed by anodic bonding of a thin glass wafer to the etched silicon wafer. The bonded wafers are then heated inside a furnace at a temperature above the softening point of the glass, and due to the expansion of the trapped gas in the silicon cavities the glass is blown into threedimensional spherical shells. An analytical model which can be used to predict the shape of the glass shells is described and demonstrated to match the experimental data. The ability to blow glass on a wafer level may enable novel capabilities including mass-production of microscopic spherical gas confinement chambers, microlenses, and complex microfluidic networks.

[2006-0092]
\end{abstract}

Index Terms-Fabrication, glass, glass blowing, glass bubble, glass shell, microglass sphere, micromachining, spheres, wafer-level manufacturing.

\section{INTRODUCTION}

$\mathbf{G}$ LASS blowing is an art that dates back over 2000 years [1]. Today, glass blowing is used in a wide array of applications, including scientific glassware, optical components, consumer glass containers, and visual arts. Although blow-molding techniques are used in the glass industry to automate the fabrication of bottles and other containers, many fine glass products are still shaped one at a time by glass blowers.

This paper introduces a fabrication process where glass is blown on a wafer level [2], [3], allowing thousands of glass parts to be built simultaneously. The presented micro glass blowing also opens opportunities for integration with electrical and mechanical components on a chip using conventional microfabrication techniques.

The fabrication process was developed for a micromachined implementation of a nuclear magnetic resonance gyroscope (NMRG), where a spherical gas confinement chamber is preferred in order to minimize the self-magnetization of the atoms. Although no previous micro-NMRGs have been reported, large NMRGs built around traditionally blown glass spheres have been demonstrated in the past (see for example [4]-[6]).

Even though not fully explored, we envision that several other novel applications will be enabled by this new fabrication technique, including mass-produced microscopic glass lenses, con-

Manuscript received May 17, 2006; revised September 30, 2006. This work is supported in part by the DARPA NGIMG program. Subject Editor F. Ayazi.

E. J. Eklund is with the Department of Electrical Engineering and Computer Science, Microsystems Laboratory, University of California, Irvine, CA 92697 , USA (e-mail: eeklund@uci.edu).

A. M. Shkel is with the Department of Mechanical and Aerospace Engineering, Microsystems Laboratory, University of California, Irvine, CA 92627 USA (e-mail: ashkel@uci.edu).

Color versions of one or more of the figures in this paper are available online at http://ieeexplore.iee.org.

Digital Object Identifier 10.1109/JMEMS.2007.892887 finement chambers for a variety of microscopic nuclear magnetic resonance devices, spacers for wafer-level packaging, and complex three-dimensional networks for gas analyzers and drug delivery systems.

The property that enables the successful shaping of glass is that its viscosity is highly dependent on the temperature. In order to shape glass it needs to be heated above its softening point, i.e., the temperature at which glass has a viscosity of $10^{6.6}$ Pascal-seconds (Pa-s) [7]. For Pyrex 7740, which is used in the process described in this paper, the softening point occurs at $821^{\circ} \mathrm{C}$ [8]. In conventional glass blowing, a gob of glass is first heated inside a furnace. The gob is then removed from the furnace and blown into desired shapes. Often the heating and blowing steps are repeated multiple times. Once the glass is shaped, it is usually annealed to remove stresses that developed during the blowing.

The original plan for process development was the direct adaptation of conventional glass blowing techniques on a microscale, i.e., to bond a glass wafer to a through-etched silicon wafer, attach a blow hose, and blow spheres when heated. However, it proved to be very difficult to create a good seal between the silicon wafer and the blow hose that could withstand the high temperatures required for glass blowing. But while pursuing this approach, multiple small bubbles were discovered in unexpected areas of the glass wafer after heating the samples. It was hypothesized that small pockets of air had been trapped between the glass and the silicon during the anodic bonding. These air pockets presumably caused the glass to deform due to the increased pressure when heated. An idea was born to try to amplify this effect by etching deep cavities in the silicon to increase the volume of the trapped air. It was later discovered that a similar process has previously been implemented to plastically deform silicon [9].

A fabricated and diced sample is shown in Fig. 1. The depicted glass-blown hollow semisphere is approximately $700 \mu \mathrm{m}$ in diameter and the chip is $2 \mathrm{~mm} \times 2 \mathrm{~mm}$.

The fabrication process is presented in Section II. Next, a three-part model of the glass blowing process is introduced in Section III. The first part of the model describes the final volume and shape of the glass shells, the second part estimates the required blow-up time, and the third part considers the nonuniform glass thickness. Section IV presents experimental results and verifies the glass blowing model by comparison to the experimental data.

\section{FABRICATION PROCESS}

In order to fabricate the glass shells a silicon wafer is first patterned with a layer of AZ P4620 photoresist, as illustrated in Fig. 2. Cylindrical cavities are then etched in the silicon wafer using timed deep reactive ion etching (DRIE). The photoresist is removed with acetone and a $100-\mu \mathrm{m}$-thin borosilicate glass 


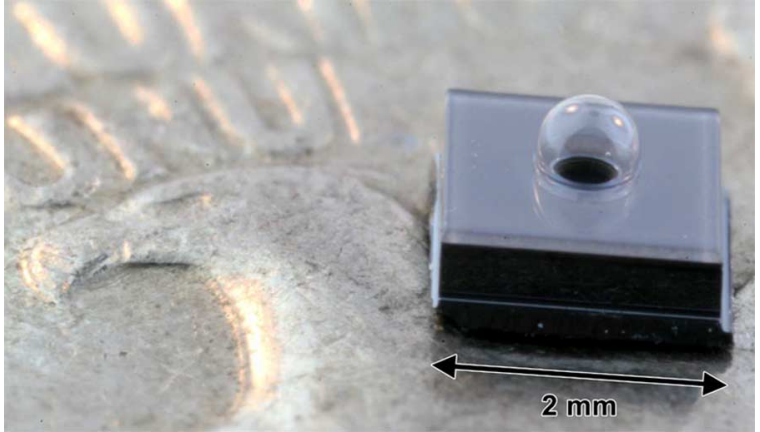

Fig. 1. Fabricated and diced chip on top of a US quarter dollar coin. The diameter of the glass-blown hollow semisphere is approximately $700 \mu \mathrm{m}$.

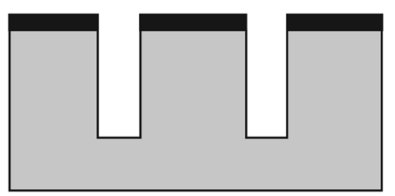

Step 1: Pattern Si wafer with photoresist and etch (DRIE)

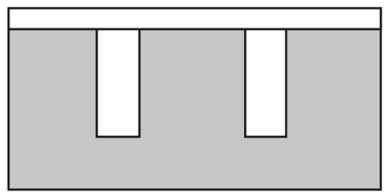

Step 2: Remove photoresist and bond thin glass wafer to Si wafer

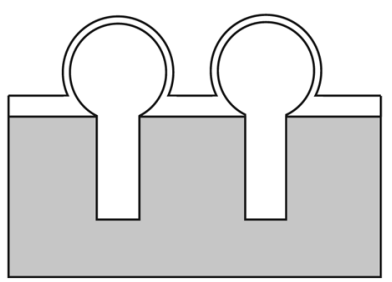

Step 3: Heat in furnace at approx. $850^{\circ} \mathrm{C}$

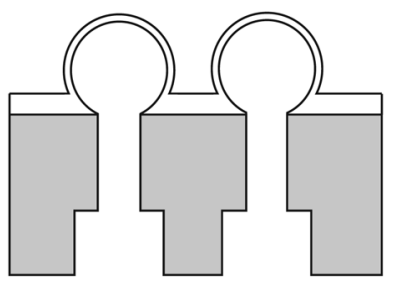

Step 4: Etch backside (OPTIONAL)

Fig. 2. Fabrication process for glass blowing on a wafer level.

wafer (Pyrex 7740) is anodically bonded to the silicon wafer, covering all of the etched cavities. Once bonded, the glass wafer may also be grinded and polished if thinner shell walls are desired.

Next, the bonded wafers are placed inside a furnace at atmospheric pressure and at a temperature above the softening point of the glass. Due to the high temperature, the pressure of the trapped air inside the sealed cavities increases and the glass deforms into spherical shapes.

The samples are quickly removed from the furnace and brought out to room temperature. The rapid removal of the glass shells is required to assure that the glass solidifies before the pressure inside the shells is reduced due to the lower temperature. If the temperature is instead allowed to slowly decrease, the glass shells will sag before they solidify and thus develop into nonspherical shapes.

Once the glass is shaped, the backside of the silicon wafer can be patterned and etched to allow for filling of various gases and other substances. The backside can then be resealed using conventional wafer bonding techniques. Note that a double-side polished silicon wafer must be used if the openings are to be resealed using anodic bonding. A holder is also needed in order to cover and protect the blown glass shells during the bonding. Etching of the backside may also be necessary if the process is used for creating microlenses or microfluidic networks.

An alternative to the etching of the backside in the last fabrication step is to fill the cavities with the desired substances before the glass is bonded in Step 2 (see Fig. 2). If this is done, the etching of the backside is not required. However, some light gases may then diffuse into or through the glass when the sample is heated inside the furnace in Step 3. Furthermore, certain substances may vaporize and increase the pressure inside the etched cavity more than desired. But for some particular substances, an additional filling step (before Step 2 ) is potentially a very appealing option when the glass shells are to be used as confinement chambers.

Another possible addition to the fabrication process is to use a mold in order to shape non-spherical structures. A wafer with predefined etched cavities can be temporarily attached on top of the glass before Step 3 in Fig. 2. For example, if cubical molds are etched in a wafer that is placed on top of the glass, hollow cubical glass structures would be obtained after the blowing in Step 3 (instead of hollow semispheres). Many other glass shapes can be made by employing molding principles. This type of blow-molding technique is similar to the processes commonly used in the glass and polymer industries to blow bottles and consumer glassware inside molds.

A final annealing step may also be added to the fabrication process. The rapid cool-down of the samples in Fig. 2 inevitably leads to residual stresses in the glass spheres. In order to remove these stresses the glass can be annealed for approximately 30 min followed by a very slow cool-down $\left(\sim 1^{\circ} \mathrm{C} / \mathrm{min}\right)$ to a temperature below the strain point of the glass. Once the temperature is well below the strain point, the cool-down rate can be increased [7]. The annealing and strain points of Pyrex 7740 are $560{ }^{\circ} \mathrm{C}$ and $510{ }^{\circ} \mathrm{C}$, respectively [8].

The modeling and experimental results in the following sections will be portrayed for the original fabrication process, which is depicted in Fig. 2.

\section{GLASS BLOWING MODEL}

The principles of the glass blowing process described in the previous section are based on the free inflation and large deformation of an initially flat glass sheet at elevated temperatures. Thus, the modeling is related to that of biaxial inflation of viscoelastic membranes, commonly used for material characterization in the polymer industry [10]-[14].

A few assumptions are made regarding the glass in order to model the fabrication process. At room temperature glass essentially behaves like an elastic solid, responding rapidly to applied stress. However at sufficiently high temperatures, stress is immediately relieved from the material due to the low viscosity of 
the glass. At high temperatures (and consequently low viscosities) glass can be modeled as a Newtonian fluid [7]. Glass also has a viscoelastic region for viscosities between approximately $10^{8} \mathrm{~Pa}$-s and $10^{13} \mathrm{~Pa}$-s.

In the fabrication process described in this paper, the glass is shaped at temperatures between $850{ }^{\circ} \mathrm{C}$ and $900{ }^{\circ} \mathrm{C}$. The viscosity in this temperature region is less than $10^{6} \mathrm{~Pa}$-s for borosilicate glass [15]. In the following sections, it is therefore assumed that the glass can be modeled as an incompressible Newtonian fluid due to the low viscosity at the elevated temperatures.

\section{A. Volume and Shape of Glass Structures}

As was illustrated in the fabrication process in Fig. 2, no external blowing is involved in the fabrication of the glass spheres. Instead, the glass components are formed by themselves due to the increased pressure inside the sealed cavities when heated. An estimate of the pressure that develops inside the cavity can be obtained from the ideal gas law

$$
P V=n R T
$$

where $P$ is the pressure, $V$ is the volume, $n$ is the number of moles, $R$ is the Boltzmann constant, and $T$ is the temperature. Since $n$ and $R$ are both constants, the ideal gas law can also be written as $P_{1} V_{1} / T_{1}=P_{2} V_{2} / T_{2}$.

The glass blowing takes place inside a furnace at atmospheric pressure, as was described in Section II. When the samples are placed inside the furnace, the high temperature will cause the pressure to increase rapidly inside the sealed cavities of the silicon wafer. At the same time the viscosity of the glass decreases and the glass sheet starts to deform. The glass will grow into a spherical shape due to the uniform pressure distribution. After a sufficiently long period of time the pressure inside the glass shells will be almost equal to the atmospheric pressure inside the furnace and most of the stresses in the glass shells will be relieved. Since the final pressure is approximately equal on the inside and the outside of the hollow semisphere and the cavities were sealed at atmospheric pressure, the ideal gas law yields the following relation between the initial volume of the etched cavity, $V_{e}$, and the volume of the blown glass shell, $V_{g}$

$$
V_{g}=V_{e}\left(\frac{T_{f}}{T_{s}}-1\right)
$$

where $T_{f}$ is the furnace temperature and $T_{s}$ is the temperature at which the cavities etched in the silicon wafer were sealed by the glass wafer.

From geometry considerations, the radius of curvature of the hollow glass semisphere develops according to

$$
r_{g}=\frac{h_{g}^{2}+r_{0}^{2}}{2 h_{g}}
$$

where $h_{g}$ is the height of the glass semisphere and it is assumed that the undeformed membrane was circular with a radius of $r_{0}$. Note that the height of the glass is measured from the bottom of

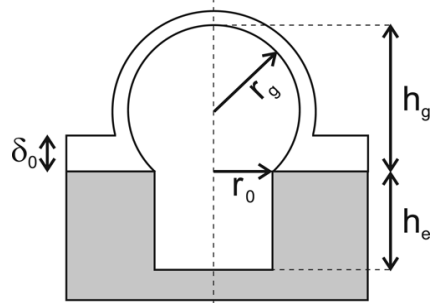

Fig. 3. Shape, height, and radius of the blown glass structures.

the undeformed glass sheet to the interior wall of the top of the blown glass shell, as illustrated in Fig. 3.

By considering the ratio between the volume of the undeformed glass membrane, $\pi r_{0}^{2} \delta_{0}$, and the approximate final volume of the glass shell, $2 \pi r_{g} h_{g} \delta$, and assuming that the glass is incompressible, the thickness of the hollow semisphere can be estimated as

$$
\delta=\frac{r_{0}^{2} \delta_{0}}{r_{0}^{2}+h_{g}^{2}}
$$

where $\delta_{0}$ is the initial thickness before the deformation. However, in reality the thickness will vary slightly over the surface of the shell with the smallest thickness at the top, as will be discussed in later sections.

In the process that was illustrated in Fig. 2 the etched cavity is cylindrical and the blown glass shell is spherical. Thus, their respective enclosed volumes are

$$
V_{e}=\pi r_{0}^{2} h_{e}
$$

and

$$
V_{g}=\frac{\pi}{3} h_{g}^{2}\left(3 r_{g}-h_{g}\right)
$$

By combining (3) and (6), the final height of the semisphere can be shown to develop as a function of the furnace temperature, the temperature at which the cavity was sealed, and the depth and radius of the etched cavity according to

$$
h_{g}=\frac{\left[\left(3 V_{g}+\sqrt{r_{0}^{6} \pi^{2}+9 V_{g}^{2}}\right) \pi^{2}\right]^{2 / 3}-r_{0}^{2} \pi^{2}}{\pi\left[\left(3 V_{g}+\sqrt{r_{0}^{6} \pi^{2}+9 V_{g}^{2}}\right) \pi^{2}\right]^{1 / 3}}
$$

where $V_{g}=h_{e} \pi r_{0}^{2}\left(T_{f} / T_{s}-1\right)$ is obtained from (2) and (5).

While it is possible to shape glass over a wide range of temperatures, empirical trials show that if the temperature is lower than $800{ }^{\circ} \mathrm{C}$ it will take a long time for the glass spheres to develop. Also, if the temperature is higher than $950{ }^{\circ} \mathrm{C}$ the spheres tend to break due to the low viscosity at higher temperatures. The best shapes were obtained at temperatures between $850{ }^{\circ} \mathrm{C}$ and $900{ }^{\circ} \mathrm{C}$ when using Pyrex 7740 borosilicate glass. The height of the semisphere as a function of the initial radius of the undeformed glass membrane (equal to the radius of the etched cavity) is plotted in Fig. 4 for etch depths of 300, 500, 


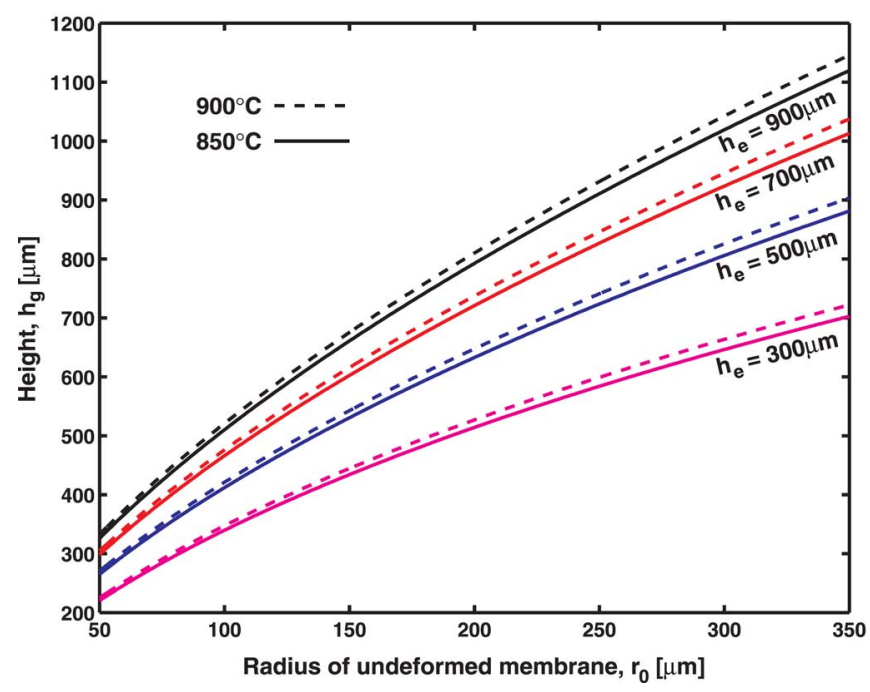

Fig. 4. Height of blown hollow semisphere as a function of the radius of the etched cavity. Plots are shown for four different etch depths and for furnace temperatures of $900{ }^{\circ} \mathrm{C}$ (dashed) and $850^{\circ} \mathrm{C}$ (solid).

700 , and $900 \mu \mathrm{m}$. Plots are shown for both $850^{\circ} \mathrm{C}$ (solid) and $900{ }^{\circ} \mathrm{C}$ (dashed).

Note that the variation in height due to furnace temperature is relatively small in the region of $850{ }^{\circ} \mathrm{C}-900{ }^{\circ} \mathrm{C}$. The radius of the etched cavity has the largest influence on the final volume of the glass shell due to the square of $r_{0}$ in (5).

In certain applications a highly spherical shape is desired. For example in a nuclear magnetic resonance gyroscope, which is the application that the wafer-level glass blowing was initially developed for, a spherical gas confinement chamber reduces the self-magnetization of the confined atoms due to symmetry. Thus, a spherical chamber can potentially improve the performance of the inertial instrument.

In order to make the shells as spherical as possible, the base radius at the bottom of the hollow semisphere should be small. Therefore it is advantageous to use thick wafers and etch deep cavities (large $h_{e}$ ) instead of increasing the etched radius $r_{0}$. The ratio between the height and the diameter of the blown semispheres, i.e., the sphericity measured in percent, is shown in Fig. 5 for different etch depths and as a function of the radius of the undeformed glass membrane.

Naturally a narrower opening, $r_{0}$, gives a more spherical shape. But even for a fairly large radius of $200 \mu \mathrm{m}$ the estimated ratio between the height and the diameter of the semisphere is greater than $90 \%$, as long as the etched cavity is deeper than $500 \mu \mathrm{m}$, as can be seen in Fig. 5 .

An alternative process, which potentially allows for larger sphericity, is illustrated in Fig. 6. In this process two silicon wafers are bonded. The first double-side polished (thin) wafer, Wafer 1, will define the base of the hollow glass semisphere and is etched all the way though with a small radius. In the second wafer, Wafer 2, a large chamber is etched. Once etched, the silicon wafers are bonded using for example a fusion bond process. Next, a thin glass wafer is anodically bonded to Wafer 1 and the sample is then placed inside a furnace in order to blow the glass. In this process the radius of the base of the glass shell, $r_{0}$, can be designed to be much smaller than the radius of the cavity etched

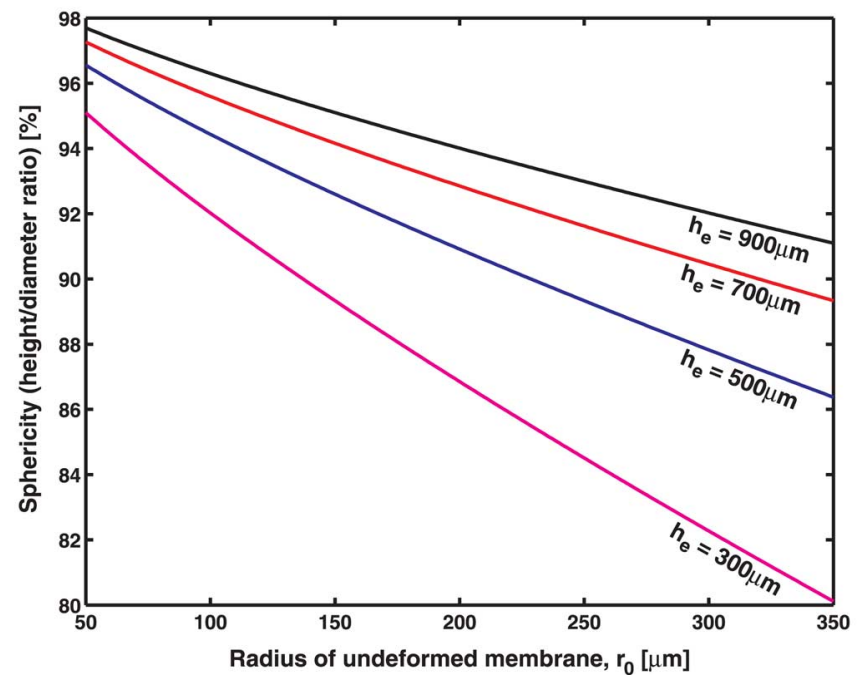

Fig. 5. Sphericity of the blown structure, i.e., the ratio between the height and the diameter of the hollow semisphere.

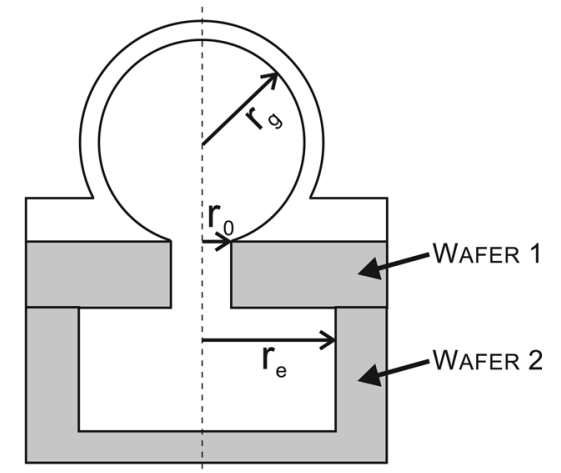

Fig. 6. Alternative fabrication process utilizing two bonded silicon wafers to achieve larger sphericity (height/diameter ratio).

in Wafer 2, $r_{e}$. While it is here assumed that the cavity etched in Wafer 2 is cylinder-shaped, only the volume matters and this wafer can readily be etched into any desired shape using either wet or dry etching. The final volume enclosed by the glass shell is determined primarily by the volume of the cavity etched in Wafer 2 and the sphericity is now independent of the cavity's radius, $r_{e}$. By utilizing this process, $r_{0}$ can be made as small as a few microns, which in turn gives a ratio between the height and the diameter of the blown hollow semisphere of close to $100 \%$, and thus potentially an almost perfect sphere.

\section{B. Required Blow-Up Time}

Consider the axisymmetric inflation of a thin circular membrane. Force equilibrium conditions lead to the following estimation of the pressure difference between the inside and outside of the resulting thin spherical membrane:

$$
\Delta P=\frac{2 \delta}{r_{g}} \sigma
$$

where $\delta$ is the thickness of the membrane, $r_{g}$ is the radius of the semisphere, and $\sigma$ is the stress (see for example [16]). A few assumptions were made during the derivations of this equation. 
First, the shell thickness is assumed to be much thinner than the radius of curvature, so stress gradients across the shell can be ignored. Furthermore, the thickness of the inflated membrane is assumed to be uniform. While this is not quite true for the described glass blowing process (as will be discussed in the next section), the above spherical shell equation can still be used to get an idea of the approximate blow-up time of the glass spheres.

As was previously discussed, the properties of the heated glass depend on the temperature. For low temperatures the glass behaves like an elastic solid, but for higher temperatures viscoelastic models are normally used. At very high temperatures glass is modeled as a Newtonian fluid [7].

Following the derivations in [17], the stress can be split into a viscoelastic part and a viscous part. The resistance to fast deformations is determined primarily by the viscous response. Now consider the top of the hollow semisphere, where the flow is purely elongational due to the biaxial stretching of the membrane. For elongational flows of a Newtonian fluid the stress is given by $\sigma=-3 \eta \dot{\epsilon}$, where $\eta$ is the viscosity and $\dot{\epsilon}$ is the strain rate [13], [18]. The strain is $\epsilon \equiv \ln \left(\delta / \delta_{0}\right)$ [19], and hence the stress can be written as

$$
\sigma=-3 \eta \frac{d}{d t}\left(\ln \frac{\delta}{\delta_{0}}\right) .
$$

In order to estimate the time required to shape the glass, (8) and (9) are combined. The height of the glass shell, $h_{g}$, now develops according to

$$
\Delta P=24 \eta r_{0}^{2} \delta_{0} \frac{h_{g}^{2}}{\left(r_{0}^{2}+h_{g}^{2}\right)^{3}} \frac{d h_{g}}{d t}
$$

where $\Delta P=P_{i}-P_{o}$ is the pressure difference between the inside, $P_{i}$, and outside, $P_{o}$, of the shell [17]. In the fabrication process presented in Section II, $P_{o}$ is equal to the furnace pressure (1 atm). The pressure inside the glass shell depends on the furnace temperature as well as the time-dependent height of the semisphere. The magnitude of this pressure was derived from the ideal gas law and the geometry considerations in the previous section as

$$
P_{i}=\frac{P_{s} \frac{T_{f}}{T_{s}}}{1+\frac{h_{g}}{6 r_{0}^{2} h_{e}}\left(h_{g}^{2}+3 r_{0}^{2}\right)}
$$

where $P_{s}$ is the pressure at which the cavities etched in the silicon wafer were sealed by the glass wafer (assumed to be $1 \mathrm{~atm}$ ). It was also assumed that the glass membrane will not significantly deform until the final temperature has been distributed uniformly throughout the chip, and thus the ideal gas law can be applied. This assumption was based on the fact that the samples are small and quickly positioned inside the furnace and should therefore heat fairly uniformly as well as rapidly. While this assumption does not quite hold true in reality, it is sufficient for the rough estimations of the order of magnitude of the blow-up time presented in this section.

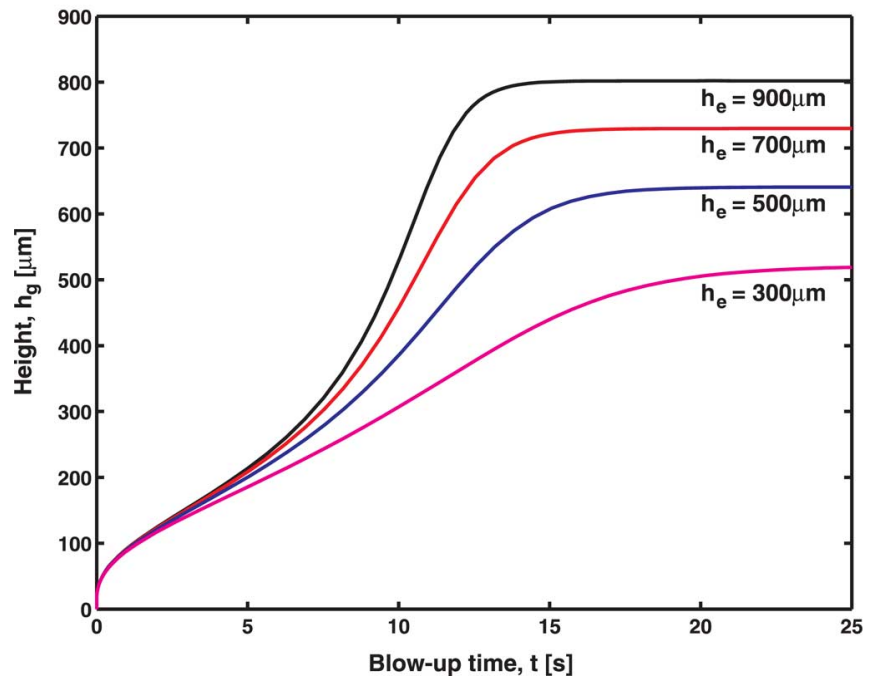

Fig. 7. Approximate time required to blow uniformly heated hollow glass semispheres at $850^{\circ} \mathrm{C}$.

As described by (10) and (11), the pressure difference, $\Delta P$, increases rapidly to a few atmospheres when the samples are placed inside the furnace. As the glass shell grows, the pressure inside the shell will decrease until it is almost equal to the pressure inside the furnace ( $1 \mathrm{~atm})$. After a sufficient period of time, the pressure difference will be close to zero.

The plot in Fig. 7 was obtained from (10) and (11). The height of the hollow glass semisphere is shown for etch depths of 300, 500, 700, and $900 \mu \mathrm{m}$. It was assumed that the etched radius, $r_{0}$, was $200 \mu \mathrm{m}$, the initial glass thickness, $\delta_{0}$, was $100 \mu \mathrm{m}$, and the viscosity of glass, $\eta$, was $10^{6} \mathrm{~Pa}$-s (approximate viscosity of borosilicate glass at $\left.850{ }^{\circ} \mathrm{C}[15]\right)$.

The blow-up time is on the order of $15 \mathrm{~s}$. Since a few extra seconds need to be added to allow for the heating of the samples, the time required to form the glass spheres inside a furnace is estimated to be on the order of one minute.

\section{Nonuniform Glass Thickness}

In the previous sections it was assumed that the thickness of the glass was uniform throughout the surface of the shell. However, due to the viscous nature of the heated glass this is not true. The top of the semisphere will be slightly thinner than the parts closer to the base. An estimate of the nonuniform wall thickness of the shell can be derived [20]

$$
\delta=\delta_{0}\left[\frac{r_{0}^{4}+r^{2} h_{g}^{2}}{r_{0}^{2}\left(r_{0}^{2}+h_{g}^{2}\right)}\right]^{2}
$$

where a particle that was initially positioned at radius $r$ before the circular membrane was deformed is considered. As the glass is blown and forms a hollow semisphere, this particle travels to a new position as shown in Fig. 8. Note that $r=0$ in the middle of the membrane, and thus the thickness of the top of the glass shell is described by $\delta=\delta_{0}\left(1+h_{g}^{2} / r_{0}^{2}\right)^{-2}$.

Depending on the particular application of the glass structures, a nonuniform wall thickness may be more or less detrimental. For some applications this property can even be utilized, 


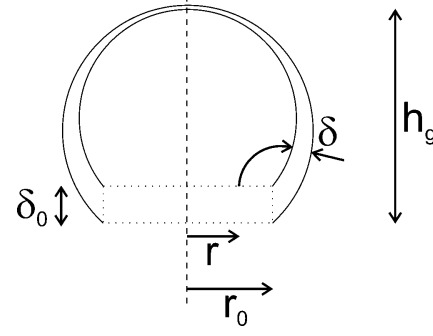

Fig. 8. Nonuniform thickness of the glass shell.

e.g., to create microlenses. The focal length of a glass shell due to the nonuniform wall thickness can be estimated from the lens makers' equation (see, for example, [21])

$$
\frac{1}{f}=\left(n_{g}-n_{0}\right)\left(\frac{1}{R_{1}}-\frac{1}{R_{2}}\right)
$$

where $R_{1}$ and $R_{2}$ are the two different meridional radii of curvature, and $n_{g}$ and $n_{0}$ are the refractive indices of the glass and the surrounding medium, respectively.

\section{EXPERIMENTAL RESULTS}

The fabrication was performed using 2-inch diameter singlecrystal silicon and Pyrex 7740 wafers. An array of cylindrical cavities was first etched in the silicon wafer using deep reactive ion etching (DRIE). Structures have been successfully fabricated for etched diameters ranging from $100 \mu \mathrm{m}$ to $1 \mathrm{~mm}$. The targeted depth of the etched cavities varied from $300 \mu \mathrm{m}$ to $800 \mu \mathrm{m}$.

Once the cavities were etched in the silicon wafer, a $100-\mu \mathrm{m}$ -thin Pyrex 7740 wafer was anodically bonded to the silicon wafer. The bonding was done at atmospheric pressure on top of a hot plate set to $400{ }^{\circ} \mathrm{C}$ and using a voltage of $600 \mathrm{~V}$.

Next the samples were diced using a diamond saw. Optionally the wafer can be diced after the blowing of the glass shells [as is shown in Fig. 9(a)], but in order to avoid potential damages to the glass structures the dicing is preferably performed before the hollow glass semispheres are blown. If the dicing is instead performed as the last fabrication step, some additional care needs to be taken in order to protect the fragile glass shells.

The samples were placed inside a furnace at temperatures ranging from $850{ }^{\circ} \mathrm{C}$ to $900{ }^{\circ} \mathrm{C}$ and at atmospheric pressure. If the samples are left for a short period of time $(\sim 1 \mathrm{~min})$ only a few of the structures are fully formed (likely due to nonuniform heating), but the shapes that do form look very spherical. Conversely if the samples are left for a longer period of time ( $\sim 5 \mathrm{~min}$ ) most of the glass shells assume a uniform shape. However, when left for too long the heated glass seems to flow down the sides of the structures (likely due to gravity) and create less spherical shapes with nonuniform shell walls. A reasonable tradeoff between shape and yield is obtained for about 3 minutes of furnace time, which was the time that the samples depicted in Fig. 9 were left inside the furnace at $850{ }^{\circ} \mathrm{C}$.

Another issue that potentially affects the final shape of the shells is the temperature used during the anodic bonding. As was shown in (7), the final height (and consequently radius) of the glass shells depends on the temperature at which the etched

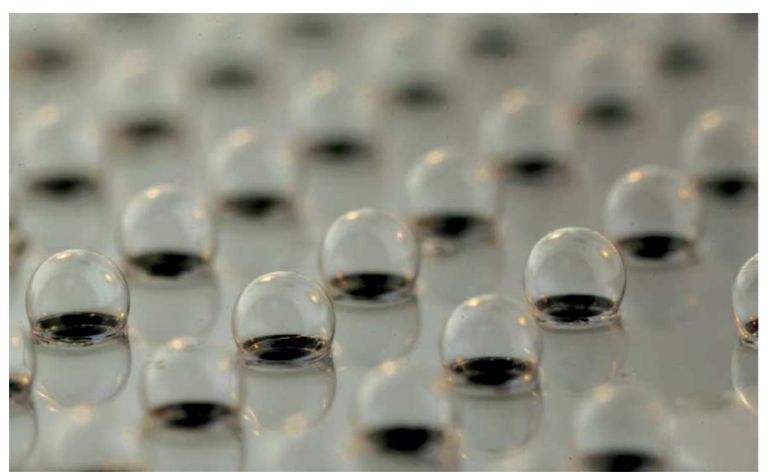

(a)

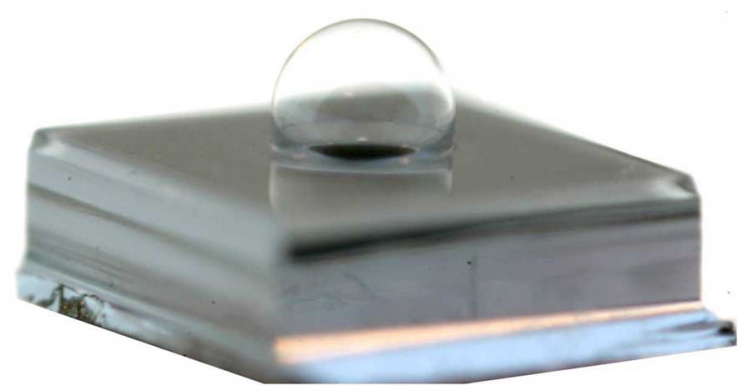

(b)

Fig. 9. Glass-blown spherical shells on a silicon wafer. The samples were formed during $3 \mathrm{~min}$ at $850^{\circ} \mathrm{C}$. The diameter of the structures is less than a millimeter. (a) Array of glass spheres. (b) Diced chip.

chambers were sealed, $T_{s}$. In the previous sections it has been assumed that $T_{s}$ is equal to room temperature. However, in order for this assumption to be valid, a sufficient force must be applied to the top electrode until the anodic bonding is completed to provide a temporary seal between the glass and the silicon. If the glass and silicon wafers are not perfectly sealed in this manner at room temperature, some air will escape from the etched cavities when heated, leading to a higher $T_{s}$ and thus a smaller final height of the glass shells. The anodic bonding can alternatively be performed inside a pressure chamber. By controlling both the temperature and the pressure during the anodic bonding, the final size of the glass shells can be accurately predicted.

Once the hollow glass spheres are fabricated, a few optional fabrication steps may be required depending on the particular application. For example, if the chambers need to be filled with gas or other substances, it may be necessary to open the backside of the wafer. The backside can be patterned and etched using either wet or dry etchants to gain access to the hollow semispheres (assuming the glass shells on the front side are protected). If double-side polished wafers are used, a rim can be maintained on the backside that will allow for resealing of the chamber using anodic bonding techniques. Other additional processing steps may include applying an anti-relaxation coating (see for example [22]), etching of the bulk glass to gain access to the silicon, and integration with other electrical and mechanical components.

\section{A. Shape of Glass Structures}

The fabricated shells in Fig. 9(a) were covered with photoresist and diced at the center of the spheres in order to be able 
TABLE I

Comparison Between the Glass Blowing Model and tHe EXPERIMENTAL Results

\begin{tabular}{|c|c|c|c|c|c|c|c|c|c|}
\hline \multirow[b]{2}{*}{ Parameter } & \multirow[b]{2}{*}{ Equation } & \multicolumn{4}{|c|}{ Chip $1\left(h_{e}=350 \mu \mathrm{m}, r_{0}=375 \mu \mathrm{m}\right)$} & \multicolumn{4}{|c|}{ Chip $2\left(h_{e}=750 \mu \mathrm{m}, r_{0}=250 \mu \mathrm{m}\right)$} \\
\hline & & Calculated & Sphere 1 & Sphere 2 & Sphere 3 & Calculated & Sphere 4 & Sphere 5 & Sphere 6 \\
\hline Glass height, $h_{g}$ & (7) & $794 \mu \mathrm{m}$ & $806 \mu \mathrm{m}$ & $834 \mu \mathrm{m}$ & $718 \mu \mathrm{m}$ & $860 \mu \mathrm{m}$ & $818 \mu \mathrm{m}$ & $814 \mu \mathrm{m}$ & $803 \mu \mathrm{m}$ \\
\hline Glass radius, $r_{g}$ & (3) & $486 \mu \mathrm{m}$ & $520 \mu \mathrm{m}$ & $540 \mu \mathrm{m}$ & $479 \mu \mathrm{m}$ & $466 \mu \mathrm{m}$ & $431 \mu \mathrm{m}$ & $439 \mu \mathrm{m}$ & $436 \mu \mathrm{m}$ \\
\hline Uniform thickness, $\delta$ & (4) & $18 \mu \mathrm{m}$ & N/A & N/A & N/A & $7.8 \mu \mathrm{m}$ & N/A & N/A & N/A \\
\hline Thickness at top, $\delta$ & (12) & $3.3 \mu \mathrm{m}$ & $14 \mu \mathrm{m}$ & $13 \mu \mathrm{m}$ & $14 \mu \mathrm{m}$ & $0.6 \mu \mathrm{m}$ & $5.3 \mu \mathrm{m}$ & $5.5 \mu \mathrm{m}$ & $7.2 \mu \mathrm{m}$ \\
\hline Thickness at side, $\delta$ & (12) & $33 \mu \mathrm{m}$ & $28 \mu \mathrm{m}$ & $22 \mu \mathrm{m}$ & $29 \mu \mathrm{m}$ & $18 \mu \mathrm{m}$ & $11 \mu \mathrm{m}$ & $12 \mu \mathrm{m}$ & $16 \mu \mathrm{m}$ \\
\hline
\end{tabular}

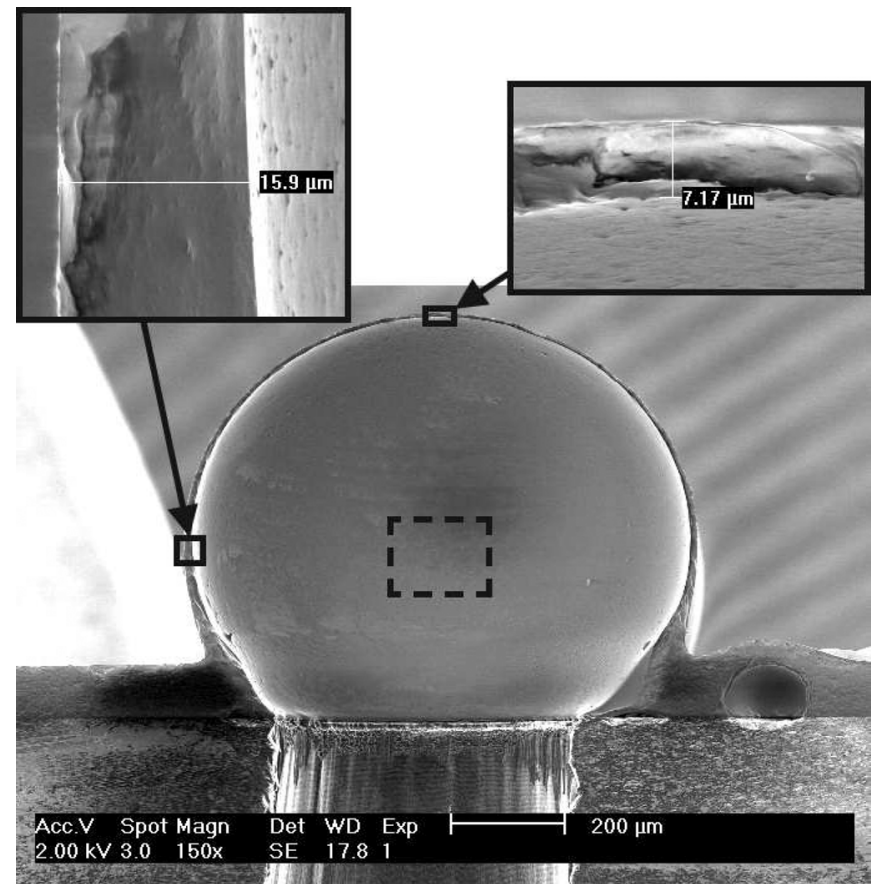

Fig. 10. Cross section of a fabricated hollow glass semisphere.

to perform metrology. A scanning electron microscope image of the cross-section of one of the hollow semispheres is shown in Fig. 10. The shell was fabricated using a $1 \mathrm{~mm}$ thick silicon wafer bonded to a $100-\mu \mathrm{m}$-thin Pyrex 7740 wafer, and was formed at $850^{\circ} \mathrm{C}$. The cylinder-shaped etched cavity is $750 \mu \mathrm{m}$ deep and $500 \mu \mathrm{m}$ in diameter.

Table I shows a comparison between the experimental results and the values predicted by the presented analytical model, calculated using the equations from the previous sections as specified in the table. Two different chips were diced and three glass shells were measured on each chip. Chip 1 was fabricated from a $450-\mu \mathrm{m}$-thick silicon wafer by etching $350-\mu \mathrm{m}$-deep cavities with a radius of $375 \mu \mathrm{m}$. A 1-mm-thick wafer was instead used to fabricate Chip 2, with an etch depth of $750 \mu \mathrm{m}$ and a radius of $250 \mu \mathrm{m}$.

The calculated height and radius agree with the experimental values in Table I. However, both (4) and (12) failed to predict the final thickness of the shells. While the thickness was not quite uniform, the thickness variation was overestimated using (12). Instead the true glass thickness was somewhere in between the thicknesses predicted by the uniform and the nonuniform models.
It should be mentioned that two other variables may have affected the results in Table I. First, while great care was taken to attempt to dice the cross-sections in the middle of the spheres, a slight offset from the center was inevitable. Therefore the actual height and radius of the glass spheres may be slightly larger than the values displayed in Table I. In addition, the specified thickness of the Pyrex 7740 wafer was $100 \mu \mathrm{m} \pm 25 \mu \mathrm{m}$. This potential variation of $50 \mu \mathrm{m}$ naturally leads to some discrepancies in the thickness results.

\section{B. Surface Quality}

The surface quality of both the inside and the outside of the side of the glass semisphere (dashed area in Fig. 10) was measured using an optical profiler (Hyphenated-Systems NanoScale 150OP). Although both surfaces were still relatively smooth, the surface roughness was greater on the outside surface. The specified initial surface roughness of the Pyrex 7740 wafers was $<10 \AA$. The average surface roughness after the spheres were formed was $2 \mathrm{~nm}$ on the inside surface and $9 \mathrm{~nm}$ on the outside. It is believed that the reason for this difference in surface roughness is that the inside surface was subjected to a uniform pressure during the blowing of the spheres, while the outside surface was directly exposed to the surrounding nitrogen gas flow and particulates inside the furnace.

\section{CONCLUSION}

A fabrication process that facilitates glass blowing on a wafer level was introduced in this paper. The presented experimental results demonstrate, for the first time, the feasibility of a parallel, wafer-level, glass-blowing process. An analytical model was also developed and shown to closely predict the shapes of the glass-blown structures.

\section{ACKNOWLEDGMENT}

The authors would like to thank the staff of the UCI Integrated Nanosystems Research Facility, INRF, for fabrication support and the members of the UCI Microsystems Laboratory and project collaborators at NIST for useful discussions.

\section{REFERENCES}

[1] A. MacFarlane and G. Martin, Glass: A World History. Chicago, IL: University of Chicago Press, 2002.

[2] E. J. Eklund and A. M. Shkel, Wafer-Level Micro-Glass-Blowing UCI Office of Technology Alliances, UC Case No, 2006-176, 2005.

[3] - Glass Blowing on a Wafer Scale (Expanded Version of 2006-176) UC Case No. 2006-519, 2006.

[4] K. F. Woodman, P. W. Franks, and M. D. Richards, "The nuclear magnetic resonance gyroscope: A review," J. Navigation, vol. 40, no. 3, pp. 366-384, 1987. 
[5] P. Härle, G. Wäckerle, and M. Mehring, "A nuclear-spin based rotation sensor using optical polarization and detection methods," Appl. Magn. Reson., vol. 5, no. 2, pp. 207-220, 1993.

[6] T. W. Kornack, R. K. Ghosh, and M. V. Romalis, "Nuclear spin gyroscope based on an atomic comagnetometer," Phys. Rev. Lett., vol. 95, no. 23, p. 230801, 2005.

[7] J. E. Shelby, Introduction to Glass Science and Technology. : The Royal Society of Chemistry, 1997.

[8] Corning, 2006, Thermal Properties of Corning Glasses [Online]. Available: http://www.corning.com/lifesciences/technical_information/techdocs/thermalprop.asp

[9] M. A. Huff, A. D. Nikolich, and M. A. Schmidt, "Design of sealed cavity microstructures formed by silicon wafer bonding," J. Microelectromech. Syst., vol. 2, no. 2, pp. 74-81, 1993.

[10] C. D. Denson and R. J. Gallo, "Measurements on the biaxial extension viscosity of bulk polymers: The inflation of a thin polymer sheet," Polym. Eng. Sci., vol. 11, no. 2, pp. 174-176, 1971.

[11] D. D. Joye, G. W. Poehlein, and C. D. Denson, "A bubble inflation technique for the measurement of viscoelastic properties in equal biaxial extensional flow," T. Soc. Rheol., vol. 16, no. 3, pp. 421-445, 1972.

[12] A. S. Wineman, "Large axisymmetric inflation of a nonlinear viscoelastic membrane by lateral pressure," T. Soc. Rheol., vol. 20, no. 2, pp. 203-225, 1976.

[13] C. J. S. Petrie, Elongational Flows. New York: Pitman, 1979.

[14] J. Rhi-Sausi and J. M. Dealy, "A biaxial extensiometer for molten plastics," Polym. Eng. Sci., vol. 21, no. 4, pp. 227-232, 1981.

[15] R. H. Doremus, Glass Science. New York: Wiley, 1973

[16] A. C. Ugural, Stresses in Plates and Shells. New York: McGraw-Hill, 1981.

[17] O. Hassager, S. B. Kristensen, J. R. Larsen, and J. Neergaard, "Inflation and instability of a polymeric membrane," J. Non-Newtonian Fluid Mech., vol. 88, no. 2, pp. 185-204, 1999.

[18] R. B. Bird, W. E. Stewart, and E. N. Lightfoot, Transport Phenomena, 2nd ed. New York: Wiley, 2001.

[19] J. M. Dealy, "Official nomenclature for material functions describing the response of a viscoelastic fluid to various shearing and extensional deformations," J. Rheol., vol. 39, no. 1, pp. 253-265, 1995.

[20] A. H. Bloksma, "A calculation of the shape of the alveograms of some rheological model substances," Cereal Chem., vol. 34, pp. 126-136, 1957.

[21] D. Malacara and Z. Malacara, Handbook of Optical Design, 2nd ed. New York: Marcel Dekker, 2004.

[22] D. Budker, L. Hollberg, D. F. Kimball, J. Kitching, S. Pustelny, and V. V. Yashchuk, "Microwave transitions and nonlinear magneto-optical rotation in anti-relaxation-coated cells," Phys. Rev. A, vol. 71, no. 1, p. 012903, 2005

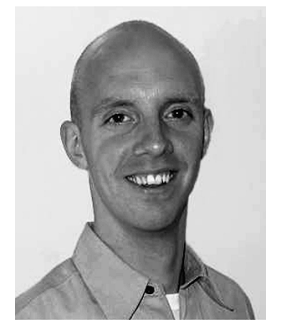

E. Jesper Eklund ( $\left.\mathrm{S}^{\prime} 05\right)$ was born in Sweden in 1979. He received the M.S. degree in electrical and computer engineering from the University of California, Irvine, in 2005, where he is currently pursuing the Ph.D. degree.

His research interests include the design and modeling of solid-state and atomic MEMS sensors. His current efforts are primarily focused on the design and fabrication of a micromachined nuclear magnetic resonance gyroscope.

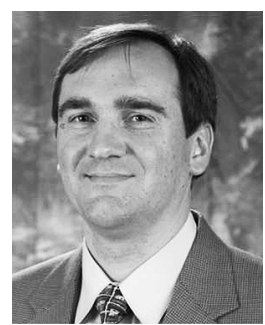

Andrei M. Shkel (S'95-A'98) received the diploma (with excellence) in mechanics and mathematics from Moscow State University, Russia, in 1991 and the $\mathrm{Ph} . \mathrm{D}$. degree in mechanical engineering from the University of Wisconsin-Madison in 1997.

He has been on the Faculty of the University of California, Irvine (UCI), since 2000, where he is an Associate Professor in the Department of Mechanical and Aerospace Engineering, in the Department of Electrical Engineering and Computer Sciences, and in the Department of Biomedical Engineering. He is also Director of the UCI Micro-Systems Laboratory. After receiving the Ph.D. degree, he joined Berkeley Sensor and Actuator Center (BSAC) at the University of California, Irvine, as a Postdoctoral Researcher. He then held research and consulting positions in several high-tech and venture companies. His professional interests, reflected in more than 100 publications, include solid-state sensors and actuators, MEMS-based neuroprosthetics, sensor-based intelligence, and control theory. He has received six U.S. and international patents (13 are pending) on micromachined angle-measuring gyroscope, wide-bandwidth rate gyroscopes, design and fabrication of light manipulators and tunable optical filters, and hybrid surface micromachining processes.

Dr. Shkel is a Member of the American Society of Mechanincal Engineering (ASME) and SPIE. He was Guest Editor for two special issues of the IEEE SENSORS JOURNAL, General Chair of the 2005 IEEE Sensors Conference, and Vice General Chair and Publications Chair of the 2002, 2003, 2004, and 2006 IEEE Sensors Conferences. He received the 2006 Research Award of the UC Irvine School of Engineering, the 2005 National Science Foundation CAREER award, the 2002 George E. Brown, Jr., Award and a 2001 Fellowship from the Japanese Advanced Science Institute. 